\title{
Password Protected Smart Card and Memory Stick Authentication against Off-Line Dictionary Attacks
}

\author{
Yongge Wang \\ UNC Charlotte, Charlotte, NC 28223, USA \\ yonwang@uncc.edu
}

\begin{abstract}
We study the security requirements for remote authentication with password protected smart card. In recent years, several protocols for passwordbased authenticated key exchange have been proposed. These protocols are used for the protection of password based authentication between a client and a remote server. In this paper, we will focus on the password based authentication between a smart card owner and smart card via an untrusted card reader. In a typical scenario, a smart card owner inserts the smart card into an untrusted card reader and input the password via the card reader in order for the smart card to carry out the process of authentication with a remote server. In this case, we want to guarantee that the card reader will not be able to impersonate the card owner in future without the smart card itself. Furthermore, the smart card could be stolen. If this happens, we want the assurance that an adversary could not use the smart card to impersonate the card owner even though the sample space of passwords may be small enough to be enumerated by an off-line adversary.
\end{abstract}

\section{Introduction}

Numerous cryptographic protocols rely on passwords selected by users (people) for strong authentication. Since the users find it inconvenient to remember long passwords, they typically select short easily-rememberable passwords. In these cases, the sample space of passwords may be small enough to be enumerated by an adversary thereby making the protocols vulnerable to a dictionary attack. It is desirable then to design password-based protocols that resist off-line dictionary attacks (see, e.g., [16]).

The problem of password-based remote authentication protocols was first studied by Gong, Lomas, Needham, and Saltzer [6] who used public-key encryption to guard against off-line password-guessing attacks. In another very influential work (see, e.g., [16]), Bellovin and Merritt introduced Encrypted Key Exchange (EKE), which became the basis for many of the subsequent works in this area. These protocols include SPEKE and SRP (see, e.g., [16]). Other papers addressing the above protocol problem can be found in [1-3]. In models discussed in the above mentioned papers, we can assume that there is a trusted client computer for the user to input her passwords. In a smart card based authentication system, this assumption may no longer be true. The smart card reader could be malicious and may intercept the user input passwords. Furthermore, a smart card could be stolen and the adversary may launch an off-line dictionary attack against the stolen smart card itself. It is the goal for this paper to discuss the security

D. Gritzalis, S. Furnell, and M. Theoharidou (Eds.): SEC 2012, IFIP AICT 376, pp. 489-500, 2012.

(C) IFIP International Federation for Information Processing 2012 
models for smart card based remote authentication and to design secure protocols within these models.

In a practical deployment of smart card based authentication systems, there may be other system requirements. For example, we may be required to use symmetric cipher based systems only or to use public key based systems. Furthermore, the system may also require that the server store some validation data for each user or the server do not store any validation (this can be considered as identity based systems). Furthermore, there may be other requirements such as user password expiration and changes.

In the following, we use an example to show the challenges in the design of secure smart card based authentication protocols. A traditional way to store or transfer the secret key for each user is to use a symmetric key cipher such as AES to encrypt user's long term secret key with user's password and store the encrypted secret key on the user's smart card or USB memory card. This will not meet our security goals against off-line dictionary attacks. For example, in an RSA based public key cryptographic system, the public key is a pair of integers $(n, e)$ and the private key is an integer $d$. With the above mentioned traditional approach, the smart card contains the value $A E S_{\alpha}(d)$ in its tamper resistant memory space, where $\alpha$ is the user's password. If such a card is stolen, the adversary could feed a message (or challenge) $m$ to the smart card for a signature. The adversary needs to input a password in order for the smart card to generate a signature. The adversary will just pick one $\alpha^{\prime}$ from her dictionary and ask the card to sign $m$. The card will "decrypt" the private key as $d^{\prime}=A E S_{\alpha^{\prime}}^{-1}\left(A E S_{\alpha}(d)\right)$ and return a signature $s^{\prime}=m^{d^{\prime}} \bmod n$ on $m$. Then the adversary only needs to check whether $s^{\prime e} \bmod n=m$. If the equation holds, the adversary knows that the guessed password is correct. That is, $\alpha^{\prime}=\alpha$. Otherwise, the attacker will remove $\alpha^{\prime}$ from the dictionary. Similar attacks work for Guillou-Quisquater (GQ), Fiat-Shamir, and Schnorr zero-knowledge identification schemes.

This example shows that the "off-line" dictionary attack in the stolen smart card environments is different from the traditional client-server based off-line dictionary attacks. There have been quite a number of papers dealing with smart card based remote authentications (see, e.g., [4, 5, 7, 10, 12, 13, 15]). However, most of these papers present attacks on protocols in previous papers and propose new protocols without proper security justification (or even a security model).

\section{Security Models}

Halevi and Krawczyk [8, Sections 2.2-2.3] introduced a notion of security for remote authentication with memorable passwords. They provide a list of basic attacks that a password-based client-server protocol needs to guard against. Though these attacks are important for password-based authentication, they are not sufficient for passwordprotected smart card based remote authentication. In the following, we provide an extended list of attacks that a password-protected smart card based authentication protocol needs to protect against. An ideal password-protected smart card protocol should be secure against these attacks and we will follow these criteria when we discuss the security of password-protected smart card authentication protocols. 
- Eavesdropping. The attacker may observe the communications channel.

- Replay. The attacker records messages (either from the communication channels or from the card readers) she has observed and re-sends them at a later time.

- Man-in-the-middle. The attacker intercepts the messages sent between the two parties (between user $\mathcal{U}$ and smart card $\mathcal{C}$ or between smart card $\mathcal{C}$ and servers $\mathcal{S}$ ) and replaces these with her own messages. For example, if she sits between the user and the smart card, then she could play the role of smart card in the messages which it displays to the user on the card reader and at the same time plays the role of users to the smart card.

- Impersonation. The attacker impersonates the user (using a stolen smart card or a fake smart card) to an actual card reader to authenticate to the remote server, impersonate a card reader to a user who inserts an authentic smart card, impersonate a card reader and a smart card (a stolen card or a fake card but without the actual user), or impersonate the server to get some useful information.

- Malicious card reader. The attacker controls the card reader and intercepts the smart card owner's input password. Furthermore, the attacker controls all of the communications between smart card and the card owner via the card reader, and all of the communications between smart card and the remote server. For example, the attacker may launch a man in the middle attack between the smart card and smart card owner.

- Stolen smart card. The attacker steals the smart card and impersonates the smart card owner to the remote server via a trusted or a malicious smart card reader. In this case, the attacker could use the stolen card to impersonate the card owner with guessed passwords to the remote server with a limited time of failures since the server may disable the card from the server side after certain number of failures. If the attacker is allowed to use the card with guessed passwords to impersonate the card owner to the remote server for unlimited times of failures, then it will be considered as an on-line dictionary attack which scenario is not considered in this paper. However, the attacker is allowed for three kinds of further attacks that we will discuss in the following. One exception that we need to make in our security model is that we will not allow the attacker to control a malicious card reader to intercept the card owner's password and then to steal the smart card. There are three kinds of attackers based on the stolen smart card scenario:

- Smart card is tamper resistant with counter protection. The attacker cannot read the sensitive information stored in the tamper resistant memory. Furthermore, the attacker may only issue a fixed amount of queries to the smart card to learn useful information. The smart card will be self-destroyed if the query number exceeds certain threshold (e.g., the GSM SIM card V2 or later has this capability).

- Smart card is tamper resistant without counter protection. The attacker cannot read the sensitive information stored in the tamper resistant memory. However, the attacker may issue large amount of queries to the smart card to learn some useful information. For example, the attacker may setup a fake server and uses a malicious card reader to guess the potential password.

- Smart card is not tamper resistant. The attacker (with the card) may be able to break the tamper resistant protection of the smart card and read the sensitive 
information stored in the tamper resistant memory. In this case, the smart card will look more like a USB memory stick that stores the user credential with password protection. But still there is a difference here. In order for the user to use USB memory stick based credentials, the user needs the access to a trusted computer to carry out the authentication. However, one may assume that even if the smart card is not tamper resistant, it is not possible for a malicious card reader to read the sensitive information on the card within a short time period (e.g., during the time that the card owner inserts the card into the card reader for an authentication).

- Password-guessing. The attacker is assumed to have access to a relatively small dictionary of words that likely includes the secret password $\alpha$. In an off-line attack, the attacker records past communications and searches for a word in the dictionary that is consistent with the recorded communications or carry out interaction with a stolen smart card without frequent server involvement (the attacker may carry out one or two sessions with server involved and all other activities without server involvement). In an on-line attack, the attacker repeatedly picks a password from the dictionary and attempts to impersonate $\mathcal{U}, \mathcal{C}, \mathcal{U}$ and $\mathcal{C}$, or $\mathcal{S}$. If the impersonation fails, the attacker removes this password from the dictionary and tries again, using a different password.

- Partition attack. The attacker records past communications, then goes over the dictionary and deletes those words that are not consistent with the recorded communications from the dictionary. After several tries, the attacker's dictionary could become very small.

We now informally sketch the definition of security models. We have three kinds of security models.

1. Type I. The attacker $\mathcal{A}$ is allowed to watch regular runs of the protocol between a smart card reader $\mathcal{R}$ (could be under the control of $\mathcal{A}$ ) and the server $\mathcal{S}$, can actively communicate with $\mathcal{R}$ and $\mathcal{S}$ in replay, impersonation, and man-in-the-middle attacks, and can also actively control a smart card reader when the card owner inserts the smart card and inputs her password. Furthermore, the attacker may steal the smart card from the user (if this happens, we assume that the attacker has not observed the user password from the previous runs of protocols) and issue a large amount of queries to the smart card using a malicious card reader. However, we assume that the smart card is tamper resistant and the attacker could not read the sensitive data from the smart card. A protocol is said to be secure in the presence of such an attacker if (i) whenever the server $\mathcal{S}$ accepts an authentication session with $\mathcal{R}$, it is the case that the actual user $\mathcal{U}$ did indeed insert her smart card into $\mathcal{R}$ and input the correct password in the authentication session; and (ii) whenever a smart card accepts an authentication session with $\mathcal{S}$, it is the case that $\mathcal{S}$ did indeed participate in the authentication session and the user $\mathcal{U}$ did indeed input the correct password.

2. Type II. The capability of the attacker is the same as in the Type I model except that when the attacker steals the smart card, it can only issue a fixed number of queries to the smart card using a malicious card reader. If the number of queries exceeds the threshold, the smart card will be self-destroyed. 
3. Type III. The capability of the attacker is the same as in the Type I model except that when the attacker steals the smart card, it will be able to read all of the sensitive data out from the smart card. But we will also assume that when a card owner inserts the card into a malicious card reader for a session of authentication, the card reader should not be able to read the information stored in the tamper resistant section of the card. In another word, the smart card is not tamper resistant only when the attacker can hold the card for a relatively long period by herself. Another equivalent interpretation of this assumption is that the attacker may not be able to intercept the password via the card reader and read the information stored in the card at the same time.

\section{Smart Card Based Secure Authentication and Key Agreement}

\subsection{Symmetric Key Based Scheme: SSCA}

In this symmetric key based smart card authentication scheme SSCA, the server should choose a master secret $\beta$ and protect it securely. Note that this master secret $\beta$ could be different for different users (cards). The Setup phase is as follows:

- For each user with identity $\mathcal{C}$ and password $\alpha$, the card maker (it knows the server's master secret $\beta$ ) sets the card secret key as $K=\mathcal{H}(\beta, \mathcal{C})$ and stores $\mathcal{K}=\mathcal{E}_{\alpha}(K)$ in the tamper resistant memory of the smart card, where $\mathcal{E}$ is a symmetric encryption algorithm such as AES and $\mathcal{H}$ is a hash algorithm such as SHA-2.

In the SSCA scheme, we assume that the smart card has the capability to generate unpredictable random numbers. There are several ways for smart card to do so. One of the typical approaches is to use hash algorithms and EPROM. In this approach, a random number is stored in the EPROM of the smart card when it is made. Each time, when a new random number is needed, the smart card reads the current random number in the EPROM and hash this random number with a secret key. Then it outputs this keyed hash output as the new random number and replace the random number content in the EPROM with this new value. In order to keep protocol security, it is important for the smart card to erase all session information after each protocol run. This will ensure that, in case the smart card is lost and the information within the tamper resistant memory is recovered by the attacker, the attacker should not able to recover any of the random numbers used in the previous runs of the protocols. It should be noted that some smart card industry uses symmetric encryption algorithms to generate random numbers. Due to the reversible operation of symmetric ciphers, symmetric key based random number generation is not recommended for smart card implementation.

Each time when the user inserts her smart card into a card reader (which could be malicious), the card reader asks the user to input the password which will be forwarded to the smart card.

1. Using the provided password $\alpha$, the card decrypts $K=\mathcal{D}_{\alpha}(\mathcal{K})$. If the password is correct, the value should equal to $\mathcal{H}(\beta, \mathcal{C})$. The card selects a random number $R_{c}$, computes $R_{A}=\mathcal{E}_{K}\left(\mathcal{C}, R_{c}\right)$, and sends the pair $\left(\mathcal{C}, R_{A}\right)$ to the card reader which will be forwarded to the server. 
2. The server recovers the value of $\left(\mathcal{C}, R_{c}\right)$ using the key $K=\mathcal{H}(\beta, \mathcal{C})$ and verifies that the identity $\mathcal{C}$ of the card is correct. If the verification passes, the server selects a random number $R_{s}$, computes $R_{B}=\mathcal{E}_{K}\left(\mathcal{C}, R_{s}\right)$, and sends $\left(\mathcal{C}, R_{B}, C_{s}\right)$ to the card reader which forwards it to the card. Here $C_{s}=\operatorname{HMAC}_{s k}\left(\mathcal{S}, \mathcal{C}, R_{s}, R_{c}\right)$ is the keyed message authentication tag on $\left(\mathcal{S}, \mathcal{C}, R_{s}, R_{c}\right)$ under the key $s k=$ $\mathcal{H}\left(\mathcal{C}, \mathcal{S}, R_{c}, R_{s}\right)$ and $\mathcal{S}$ is the server identity string.

3. The card recovers the value of $\left(\mathcal{C}, R_{s}\right)$ using the key $K=\mathcal{H}(\beta, \mathcal{C})$, computes $s k=$ $\mathcal{H}\left(\mathcal{C}, \mathcal{S}, R_{c}, R_{s}\right)$, and verifies the HMAC authentication tag $C_{s}$. If the verification passes, it computes its own confirmation message as $C_{c}=\operatorname{HMAC}_{s k}\left(\mathcal{C}, \mathcal{S}, R_{c}, R_{s}\right)$ and sends $C_{c}$ to the server. The shared session key will be $s k$.

4. The server accepts the communication if the HMAC tag $C_{c}$ passes the verification.

The protocol SSCA message flows are shown in the Figure 1.

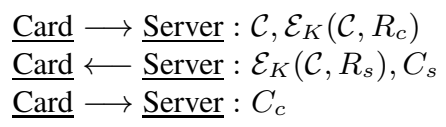

Fig. 1. Message flows in SSCA

In the following, we use heuristics to show that SSCA is secure in the Type I and Type II security models. If the underlying encryption scheme $\mathcal{E}$ and HMAC are secure, then eavesdropping, replay, man-in-the-middle, impersonation, password-guessing, and partition attacks will learn nothing about the password since no information of password is involved in these messages. Furthermore, a malicious card reader can intercept the password, but without the smart card itself, the attacker will not be able to learn information about the secret key $K=\mathcal{D}_{\alpha}(\mathcal{K})$. Thus the attacker will not be able to impersonate the server or the card owner. When the attacker steals the smart card (but she has not controlled a card reader to intercept the card owner password in the past), she may be able to insert the card into a malicious card reader and let the card to run the protocols with a fake server polynomial many times. In these protocol runs, the attacker could input guessed password $\alpha^{\prime}$. The smart card will output $\left(\mathcal{C}, \mathcal{E}_{K^{\prime}}\left(\mathcal{C}, R_{c}\right)\right)$ where $K^{\prime}=\mathcal{D}_{\alpha^{\prime}}(\mathcal{K})$. Since the attacker has no access to the actual server (this is an off-line attack), the attacker can not verify whether the output $\left(\mathcal{C}, \mathcal{E}_{K^{\prime}}\left(\mathcal{C}, R_{c}\right)\right)$ is in correct format. Thus the attacker has no way to verify whether the guessed password $\alpha^{\prime}$ is correct. In a summary, the protocol is secure in the Type I and Type II security models.

The protocol SSCA is not secure in the Type III security model. Assume that the attacker has observed a previous valid run of the protocol (but did not see the password) before steals the smart card. For each guessed password $\alpha^{\prime}$, the attacker computes a potential key $K^{\prime}=\mathcal{D}_{\alpha^{\prime}}(\mathcal{K})$. If this key $K^{\prime}$ is not consistent with the observed confirmation messages in the previous run of the protocol, the attacker could remove $\alpha^{\prime}$ from the password list. Otherwise, it guessed the correct password.

If we revise the attacker's capability in Type III model by restricting the attacker from observing any valid runs of the protocol before she steals the smart card, we get a new security model which we will call Type III' model. We can show that the protocol SSCA 
is secure in the Type III' $^{\prime}$ model. The heuristics is that for an attacker with access to the value $\mathcal{K}=\mathcal{E}_{\alpha}(K)$, he will not be able to verify whether a guessed password is valid off-line. For example, for each guessed password $\alpha^{\prime}$, she can compute $K^{\prime}=\mathcal{D}_{\alpha^{\prime}}(\mathcal{K})$. But she has no idea whether $K^{\prime}$ is the valid secret key without on-line interaction with the server. Thus the protocol is secure in the Type III' security model.

Remarks: Modification of the protocol may be necessary for certain applications. For example, if the card identification string $\mathcal{C}$ itself needs to be protected (e.g., it is the credit card number), then one certainly does not want to transfer the identification string $\mathcal{C}$ along with the message in a clear channel.

\subsection{Public Key Based Scheme: PSCAb}

In this section, we introduce a public key based smart card authentication scheme with bilinear groups: PSCAb, it is based on the identity based key agreement protocol from IEEE 1363.3 [9, 14].

In the following, we first briefly describe the bilinear maps and bilinear map groups.

1. $G$ and $G_{1}$ are two (multiplicative) cyclic groups of prime order $q$.

2. $g$ is a generator of $G$.

3. $\hat{e}: G \times G \rightarrow G_{1}$ is a bilinear map.

A bilinear map is a map $\hat{e}: G \times G \rightarrow G_{1}$ with the following properties:

1. bilinear: for all $g_{1}, g_{2} \in G$, and $x, y \in Z$, we have $\hat{e}\left(g_{1}^{x}, g_{2}^{y}\right)=\hat{e}\left(g_{1}, g_{2}\right)^{x y}$.

2. non-degenerate: $\hat{e}(g, g) \neq 1$.

We say that $G$ is a bilinear group if the group action in $G$ can be computed efficiently and there exists a group $G_{1}$ and an efficiently computable bilinear map $\hat{e}: G \times G \rightarrow G_{1}$ as above. For convenience, throughout the paper, we view both $G$ and $G_{1}$ as multiplicative groups though the concrete implementation of $G$ could be additive elliptic curve groups.

Let $k$ be the security parameter given to the setup algorithm and $\mathcal{I G}$ be a bilinear group parameter generator. We present the scheme by describing the three algorithms: Setup, Extract, and Exchange.

Setup: For the input $k \in Z^{+}$, the algorithm proceeds as follows:

1. Run $\mathcal{I} \mathcal{G}$ on $k$ to generate a bilinear group $G_{\rho}=\left\{G, G_{1}, \hat{e}\right\}$ and the prime order $q$ of the two groups $G$ and $G_{1}$. Choose a random generator $g \in G$.

2. Pick a random master secret $\beta \in Z_{q}^{*}$.

3. Choose cryptographic hash functions $\mathcal{H}_{1}:\{0,1\}^{*} \rightarrow G, \mathcal{H}_{2}:\{0,1\}^{*} \rightarrow\{0,1\}^{*}$, and $\pi: G \times G \rightarrow Z_{q}^{*}$. In the security analysis, we view $\mathcal{H}_{1}, \mathcal{H}_{2}$, and $\pi$ as random oracles.

The system parameter is $\left\langle q, g, G, G_{1}, \hat{e}, \mathcal{H}_{1}, \mathcal{H}_{2}, \pi\right\rangle$ and the master secret key is $\beta$.

Extract: For a given identification string $\mathcal{C} \in\{0,1\}^{*}$, the algorithm computes a generator $g_{\mathcal{C}}=\mathcal{H}_{1}(\mathcal{C}) \in G$, and sets the private key $d_{\mathcal{C}}=g_{\mathcal{C}}^{\beta}$ where $\beta$ is the master secret key. The algorithm will further compute $g_{\mathcal{S}}=\mathcal{H}_{1}(\mathcal{S}) \in G$ where $\mathcal{S}$ is the server 
identity string, and store the value $\left(\mathcal{C}, g_{\mathcal{S}}, d_{\mathcal{C}}^{\prime}\right)$ in the tamper resistant smart card where $d_{\mathcal{C}}^{\prime}=\mathcal{E}_{\mathcal{H}_{2}(\alpha)}\left(d_{\mathcal{C}}\right), \alpha$ is card owner's password. and $\mathcal{E}$ is the encryption function that could be defined in one of the following ways:

1. $\mathcal{E}$ is a standard symmetric cipher such as AES

2. $\mathcal{E}_{\mathcal{H}_{2}(\alpha)}\left(d_{\mathcal{C}}\right)=\operatorname{AES}_{\mathcal{H}_{2}(\alpha)}\left(d_{\mathcal{C}}\right)+i_{0}$ where $i_{0}=\min \left\{i: \operatorname{AES}_{\mathcal{H}_{2}(\alpha)}\left(d_{\mathcal{C}}\right)+i \in G, i=\right.$ $0,1, \ldots\}$. For an inputed password $\alpha^{\prime}, d_{\mathcal{C}}$ is computed as $\operatorname{AES}_{\mathcal{H}_{2}\left(\alpha^{\prime}\right)}^{-1}\left(d_{\mathcal{C}}^{\prime}-i_{0}\right)$ where $i_{0}=\min \left\{i: \operatorname{AES}_{\mathcal{H}_{2}\left(\alpha^{\prime}\right)}^{-1}\left(d_{\mathcal{C}}^{\prime}-i\right) \in G, i=0,1, \ldots\right\}$.

3. $\mathcal{E}_{\mathcal{H}_{2}(\alpha)}\left(d_{\mathcal{C}}\right)=d_{\mathcal{C}}^{\mathcal{H}_{2}(\alpha)}$

Exchange: The algorithm proceeds as follows.

1. The card selects $x \in_{R} Z_{q}^{*}$, computes $R_{A}=g_{\mathcal{C}}^{x}$, and sends it to the Server via the card reader.

2. The Server selects $y \in_{R} Z_{q}^{*}$, computes $R_{B}=g_{\mathcal{S}}^{y}$, and sends it to the card.

3. The card computes $s_{A}=\pi\left(R_{A}, R_{B}\right), s_{B}=\pi\left(R_{B}, R_{A}\right)$, and $d_{\mathcal{C}}=\mathcal{D}_{\mathcal{H}_{2}\left(\alpha^{\prime}\right)}\left(d_{\mathcal{C}}^{\prime}\right)$ where $\mathcal{D}$ is the decryption function and $\alpha^{\prime}$ is the user inputed password. If $d_{\mathcal{C}}$ is not an element of $G$, the card chooses the value for $s k$ as a random element of $G_{1}$. Otherwise, the card computes the value $s k=\hat{e}\left(g_{\mathcal{C}}, g_{\mathcal{S}}\right)^{\left(x+s_{A}\right)\left(y+s_{B}\right) \beta}$ as

$$
\hat{e}\left(d_{\mathcal{C}}^{\left(x+s_{A}\right)}, g_{\mathcal{S}}^{s_{B}} \cdot R_{B}\right) .
$$

4. The card computes $K_{1}=\mathcal{H}\left(s k, R_{A}, R_{B}, \mathcal{C}, \mathcal{S}, 1\right), K_{2}=\mathcal{H}\left(s k, R_{A}, R_{B}, \mathcal{C}, \mathcal{S}, 2\right)$, and sends $C_{\mathcal{C}}=\operatorname{HMAC}_{K_{1}}\left(\mathcal{C}, \mathcal{S}, R_{A}, R_{B}\right)$ to the server. $K_{2}$ is the shared secret.

5. The server computes $s_{A}=\pi\left(R_{A}, R_{B}\right), s_{B}=\pi\left(R_{B}, R_{A}\right)$ and $s k$ as

$$
\hat{e}\left(g_{\mathcal{C}}, g_{\mathcal{S}}\right)^{\left(x+s_{A}\right)\left(y+s_{B}\right) \beta}=\hat{e}\left(g_{\mathcal{C}}^{s_{A}} \cdot R_{A}, g_{\mathcal{S}}^{\left(y+s_{B}\right) \beta}\right) .
$$

6. The server verifies whether $C_{\mathcal{C}}$ is correct. If the verification passes, the server computes $K_{1}=\mathcal{H}\left(s k, R_{A}, R_{B}, \mathcal{C}, \mathcal{S}, 1\right), K_{2}=\mathcal{H}\left(s k, R_{A}, R_{B}, \mathcal{C}, \mathcal{S}, 2\right)$ and sends $C_{\mathcal{S}}=\mathrm{HMAC}_{K_{1}}\left(\mathcal{S}, \mathcal{C}, R_{B}, R_{A}\right)$ to the card. $K_{2}$ is the shared secret.

7. The card verifies the value of $\mathcal{C}_{\mathcal{S}}$.

The smart card should never export the value of $s k$ to the card reader during the protocol run. However, the smart card may need to export $K_{2}$ to the card reader in certain applications. The protocol PSCAb message flows are shown in the Figure 2.

In the following, we use heuristics to show that PSCAb is secure in the Type I, Type II, and Type III security models. It should be noted that if the encryption function is chosen as a standard symmetric cipher such as AES, then PSCAb is only weakly secure

$$
\begin{aligned}
& \underline{\text { Card }} \longrightarrow \underline{\text { Server }}: g_{\mathcal{C}}^{x} \\
& \underline{\text { Card }} \longleftarrow \underline{\text { Server }}: g_{\mathcal{S}}^{y} \\
& \underline{\text { Card }} \longrightarrow \underline{\text { Server }}: C_{\mathcal{C}} \\
& \underline{\text { Card }} \longleftarrow \underline{\text { Server }}: C_{\mathcal{S}}
\end{aligned}
$$

Fig. 2. Message flows in PSCAb 
in the Type III security model as follows. When the attacker has access to the value $d_{\mathcal{C}}^{\prime}$, she could remove those $\alpha^{\prime}$ from her dictionary such that $\mathcal{D}_{\mathcal{H}_{2}\left(\alpha^{\prime}\right)}\left(d_{\mathcal{C}}^{\prime}\right)$ is not an element of $G$. In other words, PSCAb is secure in the type III security model only if the remaining dictionary is still large enough.

The security of the underlying identity based key agreement protocol IDAK [14] (it is called Wang Key Agreement protocol in [9]) is proved in [14]. Furthermore, the eavesdropping, replay, man-in-the-middle, impersonation, password-guessing, and partition attacks will learn nothing about the password since no information of password is involved in these messages. Furthermore, these attackers will learn nothing about the private keys $d_{\mathcal{C}}$ and $\beta$ based on the proofs in [14]. For an attacker with access to the information $d_{\mathcal{C}}^{\prime}$ (the attacker may read this information from the stolen smart card), she may impersonate the card owner to interact with the server. Since the attacker could not compute the correct value $s k$, she will not be able to generate the confirmation message $C_{\mathcal{C}}$. Thus the server will not send the server confirmation message back to the attacker. In another word, the attacker will get no useful information for an off-line password guessing attack. Furthermore, even if the attacker has observed previous valid protocol runs, it will not help the attacker since the smart card does not contain any information of the session values $x$ of the previous protocols runs.

Remarks: In the protocol PSCAb, it is important to have the card to send the confirmation message to the server first. Otherwise, PSCAb will not be secure in the Type III security model. Assume that the server sends the first confirmation message. After the attacker obtains the value $d_{\mathcal{C}}^{\prime}$ from the smart card, she could impersonate the user by sending the vale $R_{A}$ to the server. After receiving the server confirmation message, she will remove $\alpha^{\prime}$ from her dictionary such that

$$
s k^{\prime}=\hat{e}\left(\mathcal{D}_{\mathcal{H}\left(\alpha^{\prime}\right)}\left(d_{\mathcal{C}}^{\prime}\right)^{\left(x+s_{A}\right)}, g_{\mathcal{S}}^{s_{B}} \cdot R_{B}\right)
$$

is not consistent with the confirmation message $C_{\mathcal{S}}$.

\subsection{Public Key Based Scheme: PSCA}

In the previous section, we presented a protocol PSCAb based on the identity based key agreement protocol IDAK. In this section, we briefly discuss a protocol based on the HMQV key agreement protocol [11]. Let $g$ be the generator of the group $G_{\rho}, q$ be the prime order of $g$, and $h$ be a constant. In this case, the server and the smart card will both have public keys.

The server private/public key pair is $\left(b, g^{b}\right)$. The smart card private/public key pair is $\left(a, g^{a}\right)$. The data stored on the smart card is: $\left(a \times \mathcal{H}(\alpha), g^{b}\right)$. In the following, we use $\mathcal{C}$ and $\mathcal{S}$ to denote the client (smart card) and server identity strings respectively.

1. The card selects $x \in_{R}[1, q-1]$, computes $R_{A}=g^{x}$, and sends it to the server.

2. Server selects $y \in_{R}[1, q-1]$, computes $R_{B}=g^{y}$, and sends it to the card.

3. The card decrypts the private key $a$ via the user inputed password, computes $\pi_{A}=$ $\mathcal{H}\left(R_{A}, \mathcal{S}\right), \pi_{B}=\mathcal{H}\left(R_{B}, \mathcal{C}\right), s_{A}=\left(x+\pi_{A} a\right) \bmod q$, and the shared session key: $K_{\mathrm{HMQV}}=\left(R_{B} \cdot\left(g^{b}\right)^{\pi_{B}}\right)^{s_{A} h}$. 
4. The server computes $\pi_{A}=\mathcal{H}\left(R_{A}, \mathcal{S}\right), \pi_{B}=\mathcal{H}\left(R_{B}, \mathcal{C}\right), s_{B}=\left(y+\pi_{B} b\right) \bmod q$, and the shared session key: $K_{\mathrm{HMQV}}=\left(R_{A} \cdot\left(g^{a}\right)^{\pi_{A}}\right)^{s_{B} h}$.

Remarks: Heuristics could be used to show that this protocol is secure in the Type I and Type II security models. However this protocol is not secure in the Type III security model. After the attacker obtains the value $\left(a \times \mathcal{H}(\alpha), g^{b}\right)$, the attacker could recover the password from $a \times \mathcal{H}(\alpha)$ and the smart card public key $g^{a}$. However, if $g^{a}$ is only known to the server, then PSCA should be secure in the Type III model. We conjecture that it may be impossible to design HMQV based protocols that are secure in the Type III model if the public key of the smart card is available to the attackers.

\subsection{Public Key Based Scheme with Password Validation Data at Server: PSCAV}

In previous sections, we discussed two protocols SSCA and PSCAb that the server does not store any password validation data. In this section, we discuss a protocol where the server needs to store password validation data for each card. One of the disadvantages of this kind of protocols is that if the card owner wants to change her password, the server has to be involved.

It should be noted that the password based remote authentication protocols that have been specified in the IEEE 1363.2 [9] are not secure in our models. The major reason is that the only secure credential that a client owns is the password. If the smart card owner inputs her password on an untrusted card reader, the card reader could just record the password and impersonates the client to the server without the smart card in future.

Before we present our scheme PSCAV, we briefly note that the protocol PSCAb in Section 3.2 can be easily modified to be a password protected smart card authentication scheme that the server stores user password validation data. In Section 3.2, the identity string for each user is computed as $g_{\mathcal{C}}=\mathcal{H}(\mathcal{C}) \in G$. For protocols with password validation data, we can use a different way to compute the identity strings. In particular, assume that the user $\mathcal{U}$ has a password $\alpha$, then the identity string for the user will be computed as $g_{\mathcal{C}}=\mathcal{H}(\mathcal{C}, \alpha) \in G$ and the private key for the user will be $d_{\mathcal{C}}=g_{\mathcal{C}}^{\beta}$ where $\beta$ is the master secret key. The value $\left(\mathcal{C}, g_{\mathcal{S}}, \mathcal{E}_{\mathcal{H}_{2}(\alpha)}\left(d_{\mathcal{C}}\right)\right)$ will be stored in the tamper resistant smart card, and the value $g_{\mathcal{C}}$ will be stored in the server database for this user. The remaining protocol runs the same as in Section 3.2. We can call the above mentioned protocol as PSCAbV

Now we begin to describe our main protocol PSCAV for this section. Assume that the server has a master secret $\beta$ ( $\beta$ could be user specific also). For each user with password $\alpha$, let the user specific generator be $g_{\mathcal{C}}=\mathcal{H}_{1}(\mathcal{C}, \alpha, \beta)$, the value $g_{\mathcal{C}}^{\mathcal{H}_{2}(\alpha)}$ is stored on the smart card, where $\mathcal{H}_{2}$ is another independent hash function. The value $g_{\mathcal{C}}=\mathcal{H}_{1}(\mathcal{C}, \alpha, \beta)$ will be stored in the server database for this user. The remaining of protocol runs as follows:

1. The card selects random $x$ and sends $R_{A}=g_{\mathcal{C}}^{x}$ to the server.

2. Server selects random $y$ and sends $R_{B}=g_{\mathcal{C}}^{y}$ to the card.

3. The card computes $u=\mathcal{H}\left(\mathcal{C}, \mathcal{S}, R_{A}, R_{B}\right)$ where $\mathcal{S}$ is the server identity string, $s k=g_{\mathcal{C}}^{y(x+u \alpha)}$, and sends $C_{c}=\mathcal{H}\left(s k, \mathcal{C}, \mathcal{S}, R_{A}, R_{B}, 1\right)$ to the server

4. After verifying that $C_{c}$ is correct, server computes $u=\mathcal{H}\left(\mathcal{C}, \mathcal{S}, R_{A}, R_{B}\right)$, sk= $g_{\mathcal{C}}^{y(x+u \alpha)}$, and sends $C_{s}=\mathcal{H}\left(s k, \mathcal{S}, \mathcal{C}, R_{B}, R_{A}, 2\right)$ to the card. 
The protocol PSCAV message flows are the same as for the PSCAb protocol message in the Figure 2 (but with different interpretation for the variables in the figure).

In the following, we use heuristics to show that PSCAV is secure in the Type I, Type II, and Type III security models. For the PSCAV protocol, the eavesdropping, replay, man-in-the-middle, card (client) impersonation, password-guessing, and partition attacks will learn nothing about the password due to the hardness of the Diffie-Hellman problem. For the attacker that carries out a server impersonation attack, it will receive the value $R_{A}$, and send a random $R_{B}$ to the card. The attacker will then receive the card confirmation message $C_{\mathcal{C}}$. The attacker may not launch an off-line dictionary attack on these information since for each guessed password $\alpha^{\prime}$, it has no way to generate a session key $s k^{\prime}$ due to the hardness of the Diffie-Hellman problem. For an attacker with access to the information $g_{\mathcal{C}}^{\mathcal{H}_{2}(\alpha)}$ (the attacker may read this information from the stolen smart card), she may impersonate the card owner to interact with the server. The attacker may send a random $R_{A}$ to the server which could be based on $g_{\mathcal{C}}^{\mathcal{H}_{2}(\alpha)}$, and receives a value $R_{B}$ from the server. But it cannot compute the correct value for $s k$ based on these information. Thus it could not send the confirmation message $C_{\mathcal{C}}$ to the server. Thus the server will not send the server confirmation message back to the attacker. In other words, the attacker will get no useful information for an off-line password guessing attack. Furthermore, even if the attacker has observed previous valid protocol runs, it will not help the attacker since the smart card does not contain any information of the session values $x$ of the previous protocols runs.

Remarks: The attack described in the Remarks at the end of Section 3.2 could be used to show that it is important to have the card to send the confirmation message to the server first in the protocol PSCAV also.

\section{Remote Authentication with Password Protected Portable Memory Sticks}

In this section, we investigate the scenario that the user stores her private key on a USB memory stick. Our goal is that if the memory stick is lost, then the adversary will not be able to mount an off-line dictionary attack to impersonate the legitimate user. Since a memory stick will not have its own CPU, the owner has to insert the memory stick into a trusted computer (otherwise, the malicious computer could just intercept the password and copy the content on the memory sticks and impersonates the owner in future). Thus the security model for this kind of protocols are different from the Type I, II, III models that we have discussed in Section 2, but are closely related to the Type III model. Specifically, we will have the following Type IV model for portable memory sticks.

- Type IV. The attacker $\mathcal{A}$ is allowed to watch regular runs of the protocol between a client $\mathcal{C}$ (the client will only insert her memory stick and input her password to trusted computers that are not controlled by the attacker) and the server $\mathcal{S}$, can actively communicate with $\mathcal{C}$ and $\mathcal{S}$ in replay, impersonation, and man-in-the-middle attacks, and can also steal the memory stick from the user and read the content in the memory stick. A protocol is said to be secure in the presence of such an attacker 
if (i) whenever the server $\mathcal{S}$ accepts an authentication session with $\mathcal{C}$, it is the case that the actual user $\mathcal{U}$ did indeed insert her memory stick into a computer $\mathcal{C}$ and input the correct password in the authentication session; and (ii) whenever a client $\mathcal{C}$ accepts an authentication session with $\mathcal{S}$, it is the case that $\mathcal{S}$ did indeed participate in the authentication session and the user $\mathcal{U}$ did indeed input the correct password.

Remark: It is an open question whether the security models Type III and Type IV are equivalent.

\section{References}

1. Bellare, M., Pointcheval, D., Rogaway, P.: Authenticated Key Exchange Secure against Dictionary Attacks. In: Preneel, B. (ed.) EUROCRYPT 2000. LNCS, vol. 1807, p. 139. Springer, Heidelberg (2000)

2. Boyko, V., MacKenzie, P.D., Patel, S.: Provably Secure Password-Authenticated Key Exchange Using Diffie-Hellman. In: Preneel, B. (ed.) EUROCRYPT 2000. LNCS, vol. 1807, pp. 156-171. Springer, Heidelberg (2000)

3. Bresson, E., Chevassut, O., Pointcheval, D.: Security proofs for an efficient password-based key exchange. In: ACM Conference on Computer Communications Security, pp. 241-250. ACM Press (2003)

4. Chen, Y., Chou, J., Huang, C.: Comment on four two-party authentication protocols (2010)

5. Das, M.L., Saxena, A., Gulati, V.P.: A dynamic id-based remote user authentication scheme. IEEE Transactions on Consumer Electronics 50, 629-631 (2004)

6. Gong, L., Lomas, T.M.A., Needham, R.M., Saltzer, J.H.: Protecting poorly chosen secrets from guessing attacks. IEEE J. Selected Areas in Communications 11, 648-656 (1993)

7. Goriparthi, T., Das, M.L., Saxena, A.: An improved bilinear pairing based remote user authentication scheme. Computer Standards and Interfaces 31, 181-185 (2009)

8. Halevi, S., Krawczyk, H.: Public-key cryptography and password protocols. ACM Transactions on Information and System Security 2(3), 230-268 (1999)

9. IEEE 1363. Standard specifications for public-key cryptography (2005)

10. Juang, W.S., Chen, S.T., Liaw, H.T.: Robust and efficient password-authenticated key agreement using smart cards. IEEE Trans. Industrial Electronics 55, 2551-2556 (2008)

11. Krawczyk, H.: HMQV: A High-Performance Secure Diffie-Hellman Protocol. In: Shoup, V. (ed.) CRYPTO 2005. LNCS, vol. 3621, pp. 546-566. Springer, Heidelberg (2005)

12. Lee, Y.S., Nam, J., Won, D.H.: Vulnerabilities in a Remote Agent Authentication Scheme Using Smart Cards. In: Nguyen, N.T., Jo, G.-S., Howlett, R.J., Jain, L.C. (eds.) KES-AMSTA 2008. LNCS (LNAI), vol. 4953, pp. 850-857. Springer, Heidelberg (2008)

13. Rhee, H.S., Kwon, J.O., Lee, D.H.: A remote user authentication scheme without using smart cards. Computer Standards and Interfaces 31, 6-13 (2009)

14. Wang, Y.: Efficient identity-based and authenticated key agreement protocol (2005), http://eprint.iacr.org/2005/108

15. Xiang, T., Wong, K., Liao, X.: Cryptanalysis of a password authentication scheme over insecure networks. Computer and System Sciences 74, 657-661 (2008)

16. Zhao, Z., Dong, Z., Wang, Y.: Security analysis of a password-based authentication protocol proposed to ieee 1363. Theoretical Computer Science 352, 280-287 (2006) 